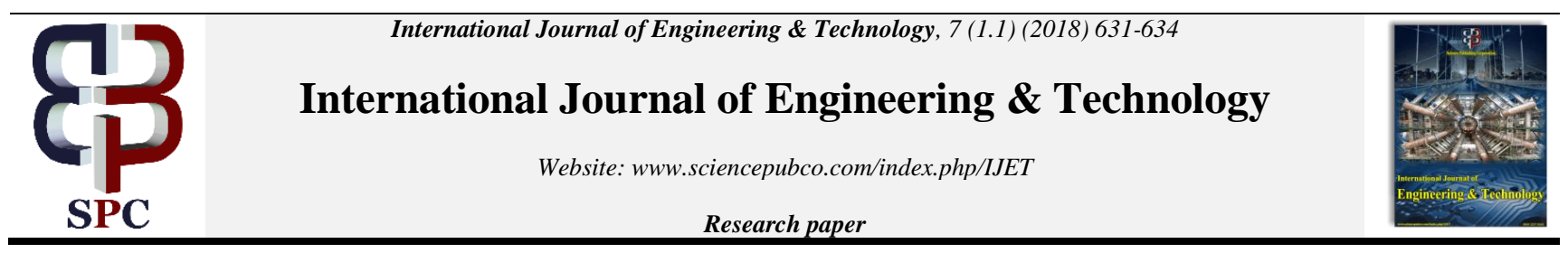

\title{
Spectral characteristics of satellite links at ka band in the southern tropical region of India
}

\author{
Sravya Velaga ${ }^{1}$, Jagadeesh Korrapati ${ }^{1}$, Chaithanya Krishna $\mathbf{B}^{1}$, Vandana Matti ${ }^{1}$, \\ K.Ch. Sri Kavya ${ }^{2}$, Sarat K. Kotamraju ${ }^{2}$ \\ ${ }^{1}$ Department of ECE, Koneru Lakshmaiah Education Foundation, Vaddeswaram, Guntur, Andhra Pradesh, India 522502 \\ ${ }^{2}$ Professor, Department of ECE, Koneru Lakshmaiah Education Foundation, Vaddeswaram, Guntur, Andhra Pradesh, India 522502 \\ *Corresponding author E-mail: kavya@kluniversity.in
}

\begin{abstract}
The objective of this study was to perform a spectral analysis of the Ka band propagation channel. Now a day, because of the expanding interest of the end users for multimedia services which require vast bandwidth, and because of the immersion of low frequency bands, for example, L, S, C bands, and $\mathrm{Ku}$ band in close future, satellite media transmission systems are moving to higher frequency bands. In such high frequency bands, the presence of the atmosphere strongly affects radiowave propagation and attenuation effects become so severe that system availability would not be sufficient if systems were designed in a conventional way with a static propagation margin.
\end{abstract}

Keywords: Rain Attenuation, Spectral Analysis, Propagation Impairments, Radiowave Propagation, Attenuation Effects.

\section{Introduction}

In the excessive worldwide growth in the use of Internet and multimedia services causes the designing for the development of broadband satellite communication systems. Due to the increasing demand of multimedia services the systems are moving towards higher frequency such as Ka band. In such high frequency bands, the presence of the atmosphere strongly affects radio-wave propagation and attenuation effects becomes severe, thus the system accessibility would not be sufficient from the user point of view if systems were designed in a very standard method with a static propagation margin [1].

One of the major drawback of the Ka band is at higher frequencies the propagation impairments become severe. For propagation attenuation free space loss is one of the important components. Atmospherical gases attenuation depends on frequency, elevation angle, and elevation higher than sea level and water vapor density. It is very negligible when compared with the rain attenuation. Another drawback of the Ka frequency band is of the increase in the signal distortion ensuing from the propagation effects and within the presence of rain the atmospherical attenuation in Ka band is often severe [2].

Thus Fade Mitigation and adaptive coding techniques to be implemented into the system so as to be able to compensate in real time propagation effects that might cause link outage. Implementing such techniques results to design a control loop that permits to insure three main functions in real time; Detection of propagation impairments, prediction of the propagation channel behavior and decision about the proper compensation to perform for the link not to be interrupted [3].

\section{Propagation Impairments}

Propagation impairments introduce the rain attenuation, cloud attenuation and scintillation. The impact of propagation impairments causes uncontrolled variations in signal amplitude, phase, polarization and angle of arrival. This ends up in a reduction with the quality of analog transmission and a rise within the error rate of digital transmission. Attenuation may be a reduction within the transmitted signal amplitude, which may degrade the accuracy and performance of the communication links. It may be caused by atmospherical gases by clouds that contain liquid water, ice particles and precipitations like rain, snow and hail[4-11].

Scintillation is the fast fluctuations of the signal parameters or radiowave like amplitude, phase, angle of arrival and polarization. For frequencies above $3 \mathrm{GHz}$, refractive index irregularities occurring within the troposphere is the main reason for the cause of tropospheric scintillation.

\section{Rain Attenuation}

Rain attenuation plays a vital role in satellite communication when the Ka frequency band is used in some the regions like tropical and sub-tropical regions. All over the world, excessive rain characteristic prediction and modelling are created. These models and predictions are statistical nature. Crane and ITU-R models are some of the common models used.

The attenuation is caused by the scattered and absorption of electromagnetic waves by drops of liquid water. The scattering decreases the signal strength and absorption creates the resonance of the waves with individual molecules and also increases the molecular energy [1], and also it results in the fall of signal energy. Attenuation is incredibly less for snow or ice crystals, because in these conditions the atoms are firmly bound and don't mix or 
combine with the waves [2]. The statistical characterization of rain starts by separating the world into rain climate zones [3]. Among the zone, the chance for a maximum rain rate is determined or decided from the data which is collected over few years.

\section{Effect of Scintillation}

Here, propagation effects are divided into two categories based upon the frequencies. They are classified as ionospheric and tropospheric. Here we can consider tropospheric scintillation because in this case study we considered frequencies more than 3 $\mathrm{GHz}$ and it contains fast and random fluctuations of rain. Therefore, Scintillation is to be determined here as tropospheric scintillation, and contains fast and random fluctuation in some of the characteristics like amplitude, polarization, phase and direction of arrival of a received signal that is resulting from the turbulence refractive index fluctuations, as a result of the turbulent mixing of air masses with various temperatures, pressure and water vapour content $[4,5]$. At low altitude angles and at higher or maximum frequencies, the scintillation might reach the value becomes equal to rain fading and impairs low margin systems. Such fast and quick fluctuations might include power management algorithms which are used for the mitigation of rain fading. For estimating and designing of the earth-space links and also the fade mitigation technique rain fading and scintillation determination is very important [6,7].

For Earth-Geo links, rain fading and the scintillation effects both are properly studied, and most of the prediction models are examined, developed and are compared with the measurement data The separation between the earth station and the satellite, and the way/path through the atmosphere don't change, which makes the computation of the attenuation is straight forward and easy/simple. The examination of rain and scintillation are equal [8-11].

\section{Spectral Analysis}

Spectral analysis is a name given to the methods of estimating the strength of various frequency elements of a time domain signal. It can be used to gauge the periodicity of a signal variation. The performance of the spectrum estimate depends on: Resolution, Variance and Bias.

A periodogram is an estimation of the spectral power of a signal. It is based on the squared magnitude of the discrete fourier transform (DFT), a mathematical procedure to determine the frequency of a discrete signal sequence [4]. If a time series has a strong sinusoidal signal for few frequencies, then there will be a peak within the periodogram at that frequency. If a time series has a strong non sinusoidal signal for few frequencies, there will be a peak in the periodogram at that frequency but also peaks at some multiples of that frequency.

Several spectral estimation techniques have been studied and issues at the form of spectrum and on computation brought about the selection of the periodogram method [5] which is based on the estimation of the auto correlation function with non-null bias.

In different rain conditions, the spectrum of the propagation channel mainly considers three slopes and it can be separated by two transition frequencies but each one is divided into separated reasons. The first slope starts from origin and it is continuous till the cut off frequency moreover it allows only slow frequency ranges because of slow phenomenon occurrence. The second zone starts from cut off frequency to transition frequency and it can separate the slow fading and fast fading components as well as it is mainly caused by rapid fluctuations inside rain, and partially by scintillation. Its slope is negative but not more than first one. The third slope starts from transition frequency to corner frequency and it is purely caused by scintillation. Fast fluctuations are the main responsible for spectral characteristics.

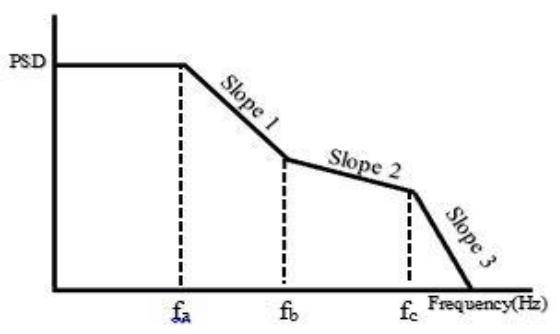

Fig.1: Theoretical Spectrum

The above figure represents the theoretical spectrum which discuss about the slopes in different rain conditions. This is plotted between the power spectral density and frequency.

Several spectral estimation methods are studied, and by considering the type of spectrum and the computation led to the choice of the periodogram method by which it relies on the estimation of the auto correlation function with non-null bias. Furthermore, the number of samples which are considered to calculate spectra was studied by considering the stationary period of the scintillation, lower than 10 minutes, of the rain duration. Estimation of the DSP from 300 samples is considered as a good trade-off between correct spectrum calculation and calculation time. In order to observe the evolution of the process during rain event, group of samples which are taken by considering certain superposition that allows a percentage of correlation between successive spectra. The next step in the process is the extraction of 5 over 6 parameters that characterize the spectrum: the three slopes and the two last frequencies of Fig.1.

An algorithm was implemented in order to select the values that best fit to the spectrum. The algorithm will use the two iterations of a function based on the least mean squares approximation. This function is used to find the slopes which will best fit the spectrum considering the non-uniform density of points by the logarithmic representation. Eventually, transition frequency and the corner frequency are found as the intersection of the two adjacent slopes.

\section{Methodology}

Here we have to calculate the power spectral density by using the different parameters and later, using power spectral values we can plot the graph between power spectral density and frequency with respect to the known data. Generally power spectral density is on FFT domain. FFT provides spectral density in the time domain mode. A periodogram is like the Fourier Transform; however it is enhanced for unevenly time-examined information and for various shapes in periodic. Here we can find the rain attenuation by using two different approaches. The first approach is about all attenuation effects being correlated and by using periodogram.

Let $\mathrm{x}_{0}, \mathrm{x}_{1}, \mathrm{x}_{2} \ldots \ldots \ldots \ldots \mathrm{x}_{\mathrm{N}-1}$ be an $\mathrm{N}$-point sequence and let

$$
\begin{array}{cc}
x[n]=x n & \text { for } n=0,1 \ldots . N-1 \\
0 & \text { Elsewhere }
\end{array}
$$

Let $X(\omega)$ be the DFT of $x[n]$.The Discrete Fourier Transform of $x_{n}$ is defined to be the $\mathrm{N}$-point sequence.

$$
\begin{aligned}
\mathrm{X}_{\mathrm{k}}= & \mathrm{X}\left(\mathrm{k} \omega_{\mathrm{s}} / \mathrm{N}\right)=\sum_{n=0}^{N-1} \mathrm{x}[\mathrm{n}] e^{-j n T k \omega \mathrm{s} / \mathrm{N}} \\
& \sum_{n=0}^{N-1} \mathrm{x}[\mathrm{n}] e^{-2 \prod j n k / \mathrm{N}} ; \mathrm{k}=0 \ldots, \mathrm{N}-1
\end{aligned}
$$

\section{Case Study}

Focusing only on the entire rain event, it is taking into account 86400 samples from June 2016 to calculate the spectrum. 


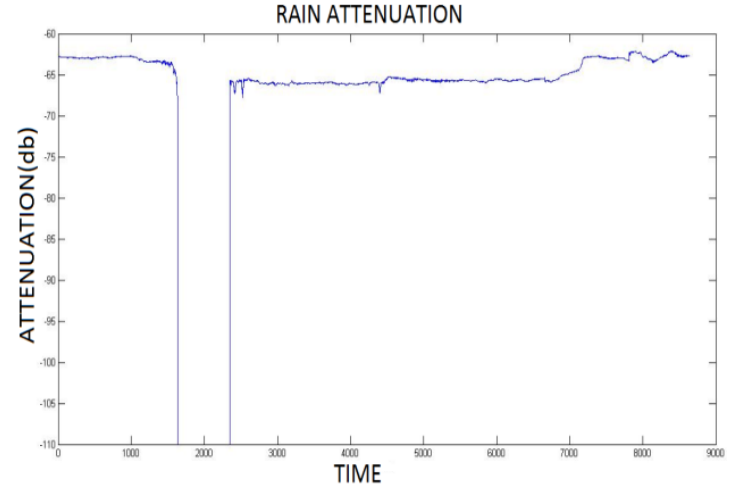

Fig.2: Rain attenuation as the function of time

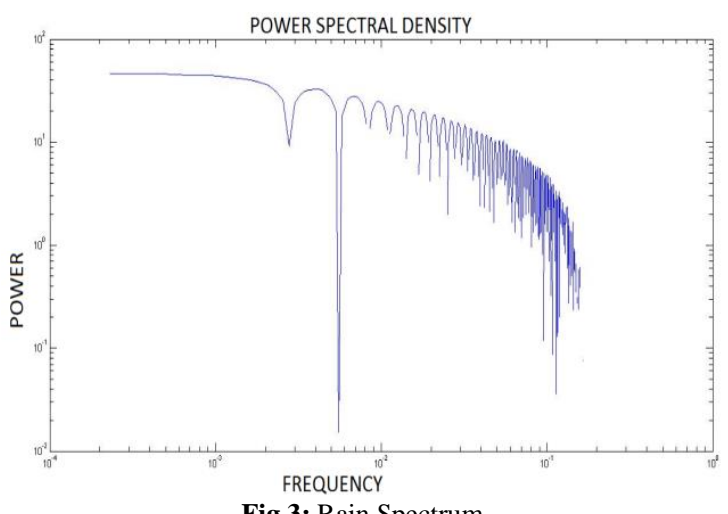

Fig.3: Rain Spectrum

These graphs (Figure 2 to7) are plotted from the reference of the graph plotted between signal level variations versus frequency. We plotted the following graphs by collecting the data of the rain occurring event on $4^{\text {th }}$ June 2016.The following graphs represent the spectra from the start to end and highest attenuation of the signal from the rain event.

Considering the clear sky situation on $4^{\text {th }}$ June 2016 at the time of a rain event, we observe the parameters that describe the spectrum. This evolution is illustrated in figures 4 to 7 , thereafter will represent the spectra from the beginning to end from the rain event of Figure2.

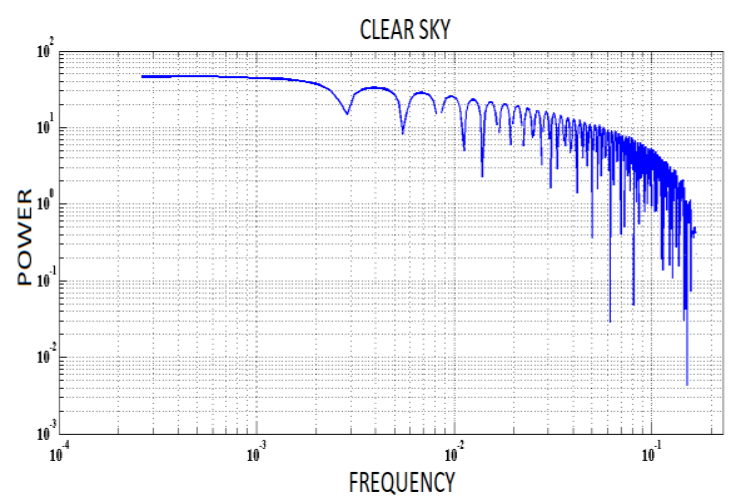

Fig.4: Clear Sky situation

In clear sky situation on $4^{\text {th }}$ June 2016 (Figure 4), the Energy of the spectrum is weak. Fast fluctuations are the main responsible for spectral characteristics.

Comparing the power spectral density during start of the event, power spectral density during end of the event plots (Figure 5 to 7 ) with the clear sky situation the energy spectrum is increased in rain occurring event i.e. on $4^{\text {th }}$ June 2016.

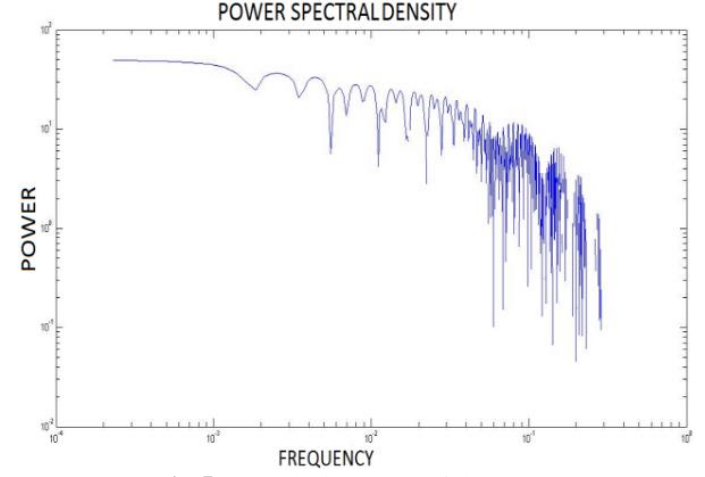

Fig.5: PSD during Start of the Event

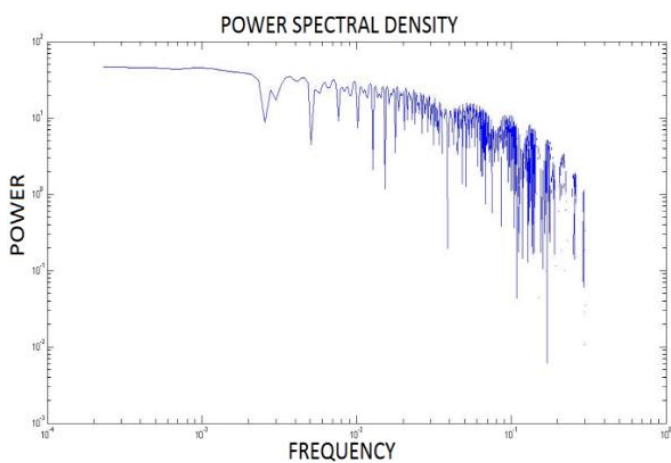

Fig. 6: PSD during End of the Event

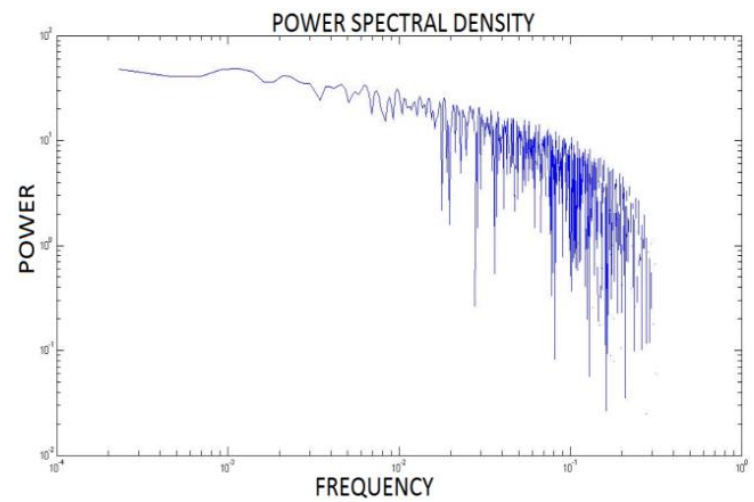

Fig 7: PSD during the event at highest attenuation of the signal.

\section{Conclusion}

The analysis of this study is to perform the spectral analysis for Ka band propagation channel as well as to monitor the status of propagation channel to know whether if an outage is occur or not, by using the control schemes to diminish the effect of outage and finally observe how the system will be going to react. It works based upon the principle of Fade mitigation technique. FMT detection function is also used to know the status of the signal however the signal is a slow varying component (caused by attenuation effects) or fast varying component (effects due to rapid fluctuations). To reach this objective, one day data has been considered on a rain occurring event and acquire the different graphs of the results give more information.

\section{Acknowledgements}

The authors especially thank the support given from Department of Science and Technology (DST), Government of India through the funded project with F. No: EMR/2015/000100. The authors also thank the management of KL University for supporting and encouraging this work by providing the facilities in Centre for Applied Research in Electromagnetics (CARE) of ECE. 


\section{References}

[1] Timothy Pratt, Charles Bostian, and Jeremy Alnutt, "Satellite communications," Wiley and sons, 2nd edition-2007.

[2] L.J.Ippolito Jr., "Satellite communications Systems Engineering," John Wiley \& sons publication, Third edition-2008.

[3] Recommendation ITU-R P.838, "Specific attenuation model for rain for use in prediction," Geneva, 2003.

[4] R. G. Lyons, Understanding digital signal processing, 2nd ed Upper Saddle River, NJ: Prentice Hall PIR, 2004.

[5] D.Vanhoenacker, A.Vander Vorst, "Atmospheric fluctuation spectra and radio system implications", ICAP 1985.

[6] L. J. Ippolito, "Propagation Effects Handbook for Satellite Systems Design," Stanford Telecom, pp. 6-102, 1999.

[7] A. D. Panagopoulos, P. D. M. Arapoglou, and P. G. Cottis, "Satellite communications at KU, KA, and V bands: Propagation impairments and mitigation techniques," Commun. Surv. Tutorials, IEEE, vol. 6, no. 3, pp. 2-14, 2004.

[8] Sarat Kumar K, "Prediction of Ku band Rain Attenuation using Experimental Data and Simulations for Hassan, India", International Journal of Computer Science and Network Security. 2008 April; 8(4).

[9] Aravind Kilaru, Sarat K Kotamraju, Nicholas Avlonitis, K.Ch. Sri Kavya, "Rain rate intensity model for communication link design across the Indian region", Journal of Atmospheric and SolarTerrestrial Physics, Volume 145, July 2016, Pages 136-142, ISSN 1364-6826, http://dx.doi.org/10.1016/ j.jastp. 2016.05.001 .

[10] John Philip, B., Kotamraju, S.K., Sri Kavya, K.C., Madhumitha, R., Pavan Kumar, A. "Performance evaluation of attenuation time series generators over Indian region" (2017) Journal of Advanced Research in Dynamical and Control Systems, 2017 (Special Issue 2), pp. 48-55.

[11] Kavya, K.C.S., Kotamraju, S.K., Charan, B.S.S.S.D., Phanindra, K., Srinivas, B., Narendra Kumar, N. "Statistical analysis of propagation parameters for fade mitigation" (2017) Journal of Theoretical and Applied Information Technology, 95 (10), pp. 2191-2196. 